\title{
Use of the COVID-19 Reporting and Data System (CO-RADS) classification and chest computed tomography involvement score (CT-IS) in COVID-19 pneumonia
}

\author{
Mehmet Özel ${ }^{1}\left[\right.$ D Aydın Aslan² ${ }^{2} \cdot$ Songül Araç ${ }^{1}$
}

Received: 25 August 2020 / Accepted: 24 January 2021 / Published online: 12 February 2021

(C) Italian Society of Medical Radiology 2021

\begin{abstract}
Purpose The increasing tendency of chest CT usage throughout the COVID-19 epidemic requires new tools and a systematic scheme for diagnosing and assessing the lung involvement in Coronavirus Disease 2019 (COVID-19). To investigate the use of the COVID-19 Reporting and Data System (CO-RADS) classification and chest CT Involvement Score (CT-IS) in COVID-19 pneumonia.

Material and methods This retrospective study enrolled 280 hospitalized patients diagnosed with COVID-19 pneumonia in a tertiary hospital in Turkey. All patients underwent non-contrast CT chest imaging. Two radiologists interpreted all CT images according to CO-RADS classification without knowing the clinical features, laboratory findings. We used CT involvement score (CT-IS) for assessing chest CT images of COVID-19 patients. Also, we examined the relationship between CT-IS and clinical outcomes in COVID-19 patients.

Results Of the patients, 111(39.6\%) had positive real-time reverse transcriptase-polymerase chain reaction (RT-PCR) results. CO-RADS 5 group patients had statistically significant positive RT-PCR results than the other groups $(P<0.001)$. All of the CO-RADS 2 group patients (30) had negative RT-PCR results. The mean total CT-IS in CO-RADS 2 group was $3.4 \pm 2.8$. The mean total CT-IS in CO-RADS 5 group was $8.2 \pm 4.7$. Total CT-IS was statistically significantly different among CORADS groups $(P<0.001)$. The mean total CT-IS was statistically significantly different between survivors and patients died of COVID-19 pneumonia $(P<0.001)$.

Conclusions CO-RADS is useful in detecting COVID-19 disease, even if RT-PCR testing is negative. CT-IS is also helpful as an imaging tool for evaluation of the severity and extent of COVID-19 pneumonia.
\end{abstract}

Keywords COVID-19 Reporting and Data System · Co-RADS · Computed tomography involvement score · COVID-19 Pneumonia

\section{Introduction}

In December 2019, a pneumonia case of unknown etiology was reported in Wuhan, which rapidly spread throughout China [1]. On January 7, 2020, this pneumonia was finally

Mehmet Özel

drmehmetozel@yahoo.com.tr

1 Department of Emergency Medicine, Diyarbakır Gazi Yasargil Training and Research Hospital, University of Health Sciences, Elazı ̆ Yolu 10. Km Üçkuyular Mevkii 21070, Kayapınar/Diyarbakır, Turkey

2 Department of Radiology, Diyarbakır Gazi Yasargil Training and Research Hospital, University of Health Sciences, Diyarbakır, Turkey found to be caused by a novel severe acute respiratory syndrome coronavirus 2 (SARS-CoV-2) [2]. A month later, the World Health Organization (WHO) named the disease as Coronavirus Disease 2019 (COVID-19) [3]. This novel coronavirus spread around the world in a short time and a pandemic is declared [3].

During the pandemic, it can be challenging to rule in/out COVID-19, in the context of enormous daily admission to the Emergency Department (ED). The real-time reverse transcriptase-polymerase chain reaction (RT-PCR) test of nasopharyngeal swab became the standard diagnostic method for COVID-19 [4]. However, in a recent study, RT-PCR positive rate was found to be between 30 and $60 \%$ at the presentation [5]. Chest CT, as a common, non-invasive diagnostic device for pneumonia, is fairly simple to perform and can 
give a rapid diagnosis. Chest $\mathrm{CT}$ takes a considerable part in the timely detection of lung infection abnormalities in the early phase for diagnosis of COVID-19 [6]. Also, recent studies have reported that chest CT could have higher sensitivity than RT-PCR in diagnosing COVID-19 [7, 8]. The Fleischner Society stated that chest CT scanning is essential in diagnosing COVID-19 patients when the symptoms worsen or RT-PCR is unavailable [9]. Moreover, chest CT scan may determine the severity of the disease based on the findings on the imagings.

CT involvement score is an imaging tool to appraise COVID-19 pneumonia. Recent studies recommended to use CT scoring criteria which also takes into account the lobe involvement in assessing COVID-19 pneumonia [10-12]. Also, in a previous study, it was reported that CT involvement score (CT-IS) can be helpful in the evaluation of the severity and extent of the COVID-19 [7].

The Dutch Radiological Society (NVvR) evolved the COVID-19 Reporting and Data System (CO-RADS) for standardization based on other trials such as Breast Imaging Reporting and Data System (BI-RADS) or Lung Imaging Reporting and Data System (Lung-RADS). CO-RADS is a categorical system to appraise the suspicion in pulmonary involvement of COVID-19 and provides standard communication on CT assessment. CO-RADS classified the pulmonary findings of COVID-19 into five levels based on $\mathrm{CT}$ findings. This classification varies from very low (CORADS 1) to very high (CO-RADS 5). Besides, a technically inadequate examination is encoding as CO-RADS 0 and if a positive RT-PCR result was proven during the examination, it encodes as CO-RADS 6 (Table 1) [8].

\section{Objectıve}

The aim of this study was to investigate the use of CORADS classification and CT-IS in COVID-19 pneumonia in the context of clinical outcomes.

\section{Methods}

\section{Setting and study design}

The institutional ethical board of the local training and research hospital reviewed and approved this retrospective study (decision date: 28 April 2020, no: 452).

\section{Patients}

This study enrolled 280 COVID-19 pneumonia patients who were diagnosed according to the WHO interim guidelines from March 20 to April 20, 2020, in the ED. Upon being diagnosed, they were admitted to the ICU or medical floor. We have obtained sociodemographic information, past medical history, clinical features, laboratory results, treatment regimen, and CT chest results by screening the electronic medical record retrospectively. All patients' outcomes were evaluated as follows: (1) patients admitted to ICU from ED; (2) patients admitted to the medical floor from ED; (3) patients were transferred to ICU from the medical floor; (4) dead during hospitalization; (5) length of hospital stay for COVID-19 pneumonia.

\section{Laboratory testing}

The nasopharyngeal swab RT-PCR assay for SARS-CoV-2 results was recorded. In addition, blood work results including complete blood count (CBC) with differential, C-reactive protein (CRP), D-dimer, lactate dehydrogenase (LDH) were tabulated.

\section{Computed tomography protocol}

All patients underwent non-contrast $\mathrm{CT}$ chest imaging in the supine position during end-inspiration on the Somatom Emotion 16-slice scanner (Siemens Healthineers, Germany). CT scan parameters: X-ray tube parameters -120 kilovoltage peak (KVp), 60 milliampere-seconds (mAs); rotation

Table 1 Overview of CO-RADS categories and the corresponding level of suspicion for pulmonary involvement in COVID-19

Level of suspicion for pulmonary involvement of Summary COVID-19

\begin{tabular}{lll}
\hline CO-RADS 0 & Not interpretable & Scan technically insufficient for assigning a score \\
CO-RADS 1 & Very low & Normal or non-infectious \\
CO-RADS 2 & Low & Typical for other infection but not COVID-19 \\
CO-RADS 3 & Equivocal/unsure & Features compatible with COVID-19, but also other diseases \\
CO-RADS 4 & High & Suspicious for COVID-19 \\
CO-RADS 5 & Very high & Typical for COVID-19 \\
CO-RADS 6 & Proven & RT-PCR positive for SARS-CoV-2 \\
\hline
\end{tabular}


time $-0.5 \mathrm{~s}$; pitch -1.0 ; section thickness $-5 \mathrm{~mm}(\mathrm{~mm})$; intersection space $-5 \mathrm{~mm}$; additionally reconstructed slice thickness of $1.5 \mathrm{~mm}$ with sharp convolution kernel.

\section{Image analysis}

Two radiologists (with 8 and 12 years of experience) interpreted all CT images according to CO-RADS classification without knowing the clinical features, laboratory findings (Table 1) [8]. Each CT chest imaging was evaluated in terms of the following characteristics: distribution of lesion, including dorsal, ventral or both dorsal and ventral lung involvement, pulmonary lobe distribution (right upper lobe (RUL), right middle lobe (RML), right lower lobe (RLL), left upper lobe (LUL), and left lower lobe (LLL)) and the whole lung; distribution of lesion including along with the peripheral area of the lung, distribution along with peribronchovascular (central) area of the lung; presence of groundglass opacity (GGO), consolidation, vascular thickening, crazy paving sign, air bronchogram, halo, reversed halo, septal thickening, pleural thickening, subpleural band, architectural distortion, vacuolization, bronchial wall thickening, centrilobular nodules; other negative findings as follows: lymphadenopathy, pleural effusion, pericardial effusion.

Also, we used CT involvement score (CT-IS) for assessing chest CT images of COVID-19 patients. Each of the 5 lung lobes was assessed for degree of involvement, such as below $5 \%$ involvement equivalent to a lobe score of 1 , 5-25\% involvement to a lobe score of 2, 26-49\% involvement to a lobe score of 3,50-75\% involvement to a lobe score of 4 , and above $75 \%$ involvement to a lobe score of 5 . A whole lung CT-IS total CT-IS" was met by aggregating 5 lobe scores (range of scores, 1-25).

\section{Statistical analysis}

The SPSS version 22.0 (IBM SPSS Statistics for Windows, version 22.0. Armonk, United States of America) was used for statistical analysis. The normality of data was tested by using the Kolmogorov Smirnov test, continuous variables were compared with the Kruskal-Wallis and Mann-Whitney $\mathrm{U}$ test, and categorical variables were compared using the chi-square test. The Dunn Bonferroni test was used for post hoc comparisons in values that were found to be significant according to the Kruskal-Wallis test. A $P$ value of $<0.05$ was considered as significant.

\section{Results}

This study recruited 280 (130 women and 150 men) COVID19 pneumonia patients. Demographic data, vital parameters, presence of comorbidities, outcomes of patients with
COVID-19 pneumonia, and the patient's distribution of CORADS groups were displayed in Table 2 . The mean age was $45.9 \pm 15.9$ years with ranging from 18 to 91 year old.

All initial laboratory test results were compared with CO-RADS groups (Table 3). D-dimer, platelet lymphocyte rate, lymphocyte count, white blood cell count (WBC), CRP, LDH, parameters were statistically significant different among CO-RADS groups $(P=0.016, P=0.019, P<0.001$, for the rest parameters).

CT-IS of all chest CT images were calculated and compared with CO-RADS groups (Table 3). The mean total CT-IS was $6.3 \pm 4.7$ in all patients. The mean total CT-IS in CO-RADS 2 group was $3.4 \pm 2.8$. The mean total CT-IS in CO-RADS 5 group was $8.2 \pm 4.7$. Total CT-IS was statistically significantly different among CO-RADS groups $(P<0.001)$. In subgroup analyses, the mean total CT-IS in CO-RADS 5 group was statistically significantly higher than the other CO-RADS groups ( $P<0.001$, all subgroups). Also, the mean total CT-IS in CO-RADS 4 group was statistically higher than CO-RADS 3 group ( $P=0.011$ ) (Figs. 1b, 2).

All chest CT features were shown in Table 4. The extension of pulmonary lesion involvement in the CO-RADS 5 group was statistically significantly greater than the other CO-RADS groups $(P<0.001$, for each lobe involvement). Ventral involvement in the CO-RADS 2 group was significantly greater than the other groups $(P=0.01)$ (Fig. 1a). Dorsal involvement in the CO-RADS 5 group was statistically significantly greater than the other groups $(P<0.001)$. CORADS 5 group had statistically significantly greater RLL (dominant lobe)involvement than the others $(P<0.001)$. Of patients, 49 (17.5\%) had both dominant lobe involvement. in CO-RADS 5 group had statistically significantly greater RLL + LLL (multi dominant lobe) involvement than the other groups $(P=0.03)$ (Fig. 3). The distribution of pulmonary lesions in the CO-RADS 2 group patients found to have statistically significantly greater peribronchovascular (central) involvement than the other groups $(P=0.002)$, also in CO-RADS group 4 and 5 were found to have statistically significantly greater peripheral involvement than the other groups $(P<0.001)$. The presence of GGO, crazy paving sign, vascular thickening sign, halo sign, subpleural band, architectural distortion, vacuolization, in the CO-RADS 5 group, was statistically significantly greater than the other groups ( $P<0.001$, for each comparison) (Fig. 3). The presence of crazy paving sign, vascular thickening sign, was not found in CO-RADS 2 group. Also, the presence of tree in bud, centrilobular nodules, and bronchial wall thickening in the CO-RADS 2 group was statistically significantly greater than the other groups (both $P<0.001$ ).

Of the patients, 111 (39.6\%) had positive RT-PCR results. CO-RADS 5 group patients had statistically significantly higher positive RT-PCR than the other groups $(P<0.001)$. All CO-RADS 2 group patients had negative RT-PCR 
Table 2 Demographic data, vital parameters, presence of comorbidities and outcomes between COVID-19 Reporting and Data System (CORADS) groups

\begin{tabular}{|c|c|c|c|c|c|c|}
\hline & Total $(N: 280)$ & $\begin{array}{l}\text { CO-RADS } 2 \\
\text { group }(N: 30)\end{array}$ & $\begin{array}{l}\text { CO-RADS } 3 \\
\text { group }(N: 42)\end{array}$ & $\begin{array}{l}\text { CO-RADS } 4 \\
\text { group }(N: 30)\end{array}$ & $\begin{array}{l}\text { CO-RADS } 5 \\
\text { group }(N: 178)\end{array}$ & $P^{*}$ \\
\hline Age (years) & $45.9 \pm 15.9$ & $39.0 \pm 14.6$ & $40.1 \pm 16.2$ & $45.1 \pm 16.3$ & $48.6 \pm 15.3^{\mathrm{AB}}$ & $<0.001$ \\
\hline $\operatorname{Sex}(N, \%)$ & & & & & & 0.202 \\
\hline Female & $130(46.4)$ & $15(50)$ & $20(47.6)$ & $19(63.3)$ & $76(42.7)$ & \\
\hline Male & $150(53.6)$ & $15(50)$ & $22(52.4)$ & $11(36.7)$ & $102(57.3)$ & \\
\hline Hypertension $(N, \%)$ & $44(15.7)$ & $3(10)$ & $6(14.3)$ & $5(16.7)$ & $30(16.9)$ & 0.801 \\
\hline Diabetes $(N, \%)$ & $36(12.9)$ & $2(6.7)$ & $3(7.1)$ & $5(16.7)$ & $26(14.6)$ & 0.373 \\
\hline COPD-asthma $(N, \%)$ & $16(5.7)$ & $3(10.0)$ & $1(2.4)$ & $2(6.7)$ & $10(5.6)$ & 0.584 \\
\hline Cardiovascular disease $(N, \%)$ & $25(8.9)$ & $4(13.3)$ & $1(2.4)$ & $1(3.3)$ & $19(10.7)$ & 0.191 \\
\hline Chronic kidney disease $(N, \%)$ & $4(1.4)$ & $0(0.0)$ & $2(4.8)$ & $0(0.0)$ & $2(1.1)$ & 0.231 \\
\hline Demans & $3(1.1)$ & $1(3.3)$ & $1(2.4)$ & $0(0.0)$ & $1(0.6)$ & 0.409 \\
\hline Other comorbidities $(N, \%)$ & $8(2.9)$ & $0(0.0)$ & $3(7.1)$ & $1(3.3)$ & $4(2.2)$ & 0.27 \\
\hline Duration of symptom (days) & $4.8 \pm 2.7$ & $5.7 \pm 3.5$ & $4.1 \pm 2.5$ & $4.7 \pm 2.8$ & $4.7 \pm 2.6$ & 0.226 \\
\hline Systolic BP (mmHg) & $116.3 \pm 13.6$ & $114.1 \pm 14.7$ & $113.2 \pm 14.5$ & $115.2 \pm 18.3$ & $117.7 \pm 12.1$ & 0.006 \\
\hline Diastolic BP (mmHg) & $71.8 \pm 8.2$ & $70.1 \pm 7.1$ & $70.6 \pm 8.4$ & $70.2 \pm 9.6$ & $72.7 \pm 8.0$ & 0.072 \\
\hline Fever $\left({ }^{0} \mathrm{C}\right)$ & $37.1 \pm 0.6$ & $36.8 \pm 0.5$ & $37.1 \pm 0.7$ & $37.4 \pm 0.6^{\mathrm{A}}$ & $37.1 \pm 0.5^{\mathrm{C}}$ & 0.004 \\
\hline Pulse (per min.) & $85.2 \pm 11.5$ & $87.6 \pm 9.5$ & $84.6 \pm 8.8$ & $90.3 \pm 15.4$ & $84.0 \pm 11.5$ & 0.057 \\
\hline Length of stay (days) & $9.2 \pm 6.1$ & $7.6 \pm 3.8$ & $9.3 \pm 7.7$ & $9.0 \pm 3.7$ & $9.5 \pm 6.3$ & 0.057 \\
\hline Hospitalization $(N, \%)$ & & & & & & 0.179 \\
\hline Clinic & $248(88.6)$ & $29(96.7)$ & $37(88.1)$ & $28(93.3)$ & $154(86.5)$ & \\
\hline ICU & $14(5.0)$ & $0(0.0)$ & $0(0.0)$ & $1(3.3)$ & $13(7.3)$ & \\
\hline ICU transfer & $18(6.4)$ & $1(3.3)$ & $5(11.9)$ & $1(3.3)$ & $11(6.2)$ & \\
\hline Outcomes & & & & & & 0.222 \\
\hline Survival & $262(93.6)$ & $30(100.0)$ & $41(97.6)$ & $28(93.3)$ & $163(91.6)$ & \\
\hline Death during hospitalization & $18(6.4)$ & $0(0.0)$ & $1(2.4)$ & $2(6.7)$ & $15(8.4)$ & \\
\hline
\end{tabular}

Data are mean (standard deviation-SD) or $N(\%)$. Data are represented as mean values \pm SD for continuous variables and as number (percentage) for categorized variables. Post hoc Dunn-Bonferroni test used on parameters with $P<0.05$

*Kruskal-Wallis and chi-square test; ${ }^{\mathrm{A}}<0.01$ versus CO-RADS 2 group; ${ }^{\mathrm{B}}<0.01$ versus CO-RADS 3 group; ${ }^{\mathrm{C}}<0.05$ versus CO-RADS 2 group; $C O P D$ chronic obstructive pulmonary disease, $B P$ blood pressure, min. minutes; $I C U$ intensive care unit

Table 3 Computed tomography involvement score (CT -IS) and laboratory findings between COVID-19 Reporting and Data System (CORADS) groups

\begin{tabular}{lcccccc}
\hline & Total $(N: 280)$ & $\begin{array}{c}\text { CO-RADS 2 } \\
\text { group }(N: 30)\end{array}$ & $\begin{array}{c}\text { CO-RADS 3 } \\
\text { group }(N: 42)\end{array}$ & $\begin{array}{l}\text { CO-RADS 4 } \\
\text { group }(N: 30)\end{array}$ & CO-RADS 5 group (N: 178) & $P^{*}$ \\
\hline CT-IS & $6.3 \pm 4.7$ & $3.4 \pm 2.8$ & $1.9 \pm 1.2$ & $4.1 \pm 2.7^{\mathrm{A}}$ & $8.2 \pm 4.7^{\mathrm{BCD}}$ & $<0.001$ \\
WBC & $7.4 \pm 4.6$ & $10.4 \pm 6.2$ & $7.6 \pm 3.4$ & $7.6 \pm 4.6^{\mathrm{E}}$ & $6.8 \pm 4.5^{\mathrm{B}}$ & 0.009 \\
NEU & $5.1 \pm 3.4$ & $7.7 \pm 6.0$ & $5.1 \pm 3.2$ & $5.3 \pm 4.3$ & $4.6 \pm 2.4^{\mathrm{F}}$ & 0.001 \\
LYM & $1.7 \pm 2.7$ & $1.9 \pm 0.8$ & $1.9 \pm 0.8$ & $1.6 \pm 0.6$ & $1.7 \pm 3.3^{\mathrm{EG}}$ & 0.121 \\
PLT & $231.0 \pm 79.6$ & $242.5 \pm 74.8$ & $235.1 \pm 73.2$ & $253.4 \pm 90.4$ & $224.3 \pm 79.5$ & 0.019 \\
PLR & $166.2 \pm 89.9$ & $145.5 \pm 75.4$ & $145.6 \pm 84.8$ & $170.8 \pm 65.7$ & $173.8 \pm 95.9$ & $<3.9$ \\
CRP & $46.2 \pm 63.8$ & $51.4 \pm 87.7$ & $22.1 \pm 46.7$ & $32.4 \pm 49.5$ & $53.4 \pm 63.4^{\mathrm{C}}$ & $<0.001$ \\
LDH & $281.8 \pm 128.2$ & $243.7 \pm 92.4$ & $221.5 \pm 87.6$ & $219.5 \pm 67.1$ & $313.0 \pm 1388^{\mathrm{CFH}}$ & $<0.001$ \\
D-dimer & $331.6 \pm 506.5$ & $279.8 \pm 342.6$ & $261.7 \pm 483.6$ & $410.0 \pm 530.8$ & $343.7 \pm 531.1^{\mathrm{A}}$ & 0.016 \\
\hline
\end{tabular}

*Kruskal-Wallis; ${ }^{\mathrm{A}}<0.05$ versus CO-RADS 3 group; ${ }^{\mathrm{B}}<0.001$ versus CO-RADS 2 group; ${ }^{\mathrm{C}}<0.001$ versus CO-RADS 3 group; ${ }^{\mathrm{D}}<0.001$ versus CO-RADS 4 group; ${ }^{\mathrm{E}}<0.05$ versus CO-RADS 2 group; ${ }^{\mathrm{F}}<0.01$ versus CO-RADS 2 group; ${ }^{\mathrm{G}}<0.01$ versus CO-RADS 3 group; ${ }^{\mathrm{H}}<0.001$ versus CO-RADS 4 group; WBC: white blood cell $\left(4000-10,000 / \mathrm{mm}^{3}\right), \mathrm{NEU}=$ neutrophil $\left(2000-7000 / \mathrm{mm}^{3}\right), \mathrm{LYM}=1 \mathrm{ymphocyte}\left(800-4000 / \mathrm{mm}^{3}\right)$, $\mathrm{PLT}=$ platelet $\left(150,000-450,000 / \mathrm{mm}^{3}\right) ; \mathrm{CRP}=\mathrm{C}$-reactive protein $(0-5 \mathrm{mg} / \mathrm{L}) ; \mathrm{LDH}=$ lactate dehydrogenase $(135-225 \mathrm{U} / \mathrm{l}) ; \mathrm{D}-\mathrm{dimer}(\mathrm{mg} / \mathrm{L})$

Post hoc Dunn-Bonferroni test used on parameters with $P<0.05$ 

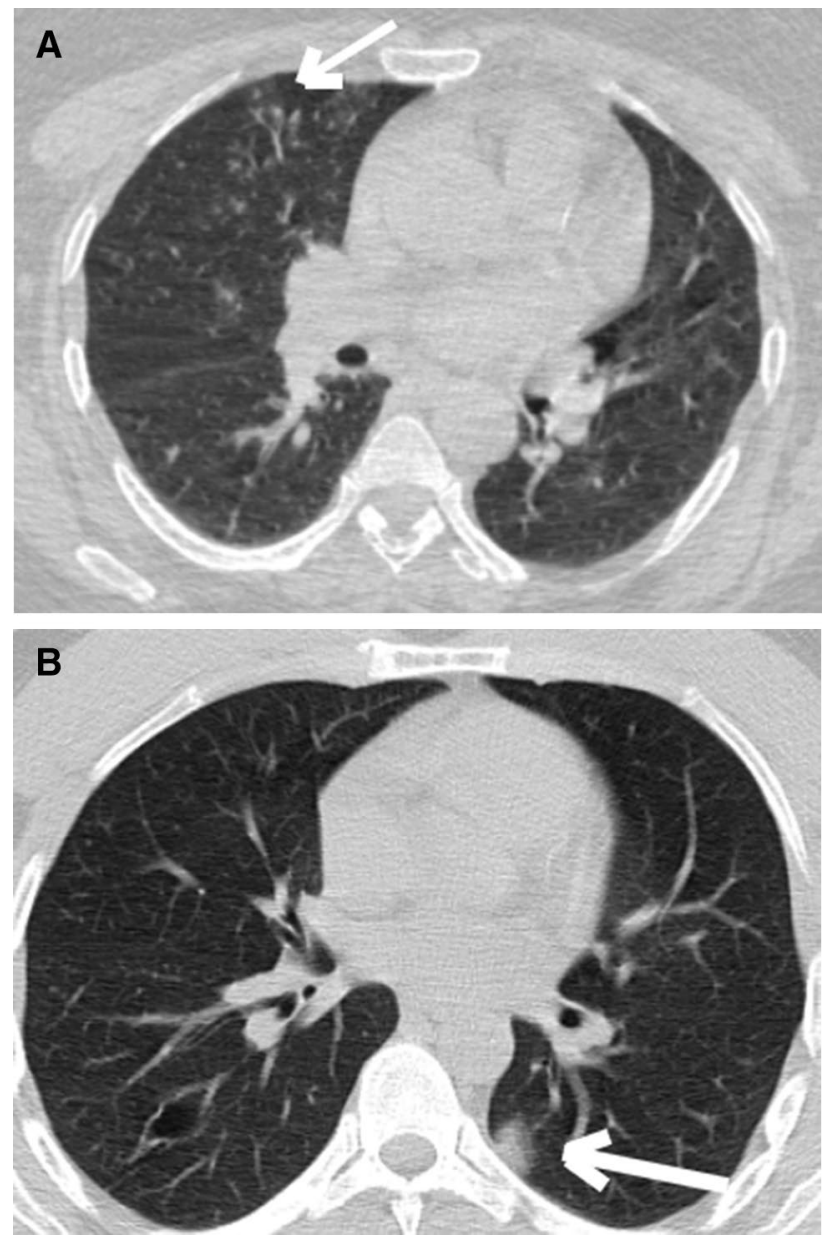

Fig. 1 a CO-RADS 2: CT scans of 52-year old female with COVID19 Centrilobular nodular and tree in bud in the right lung middle lob (white arrow). b CO-RADS 3: CT scans of 41-year old male with COVID-19 Left lung, lower lob, dorsal distribution, unifocal peripheral ground-glass opacities (GGO)



Fig. 2 CO-RADS 4: CT Scan of 33-year old female with COVID-19 Right lung, lower lob, dorsal distribution, unilateral peripheral carzy paving patern (thickened interlobuler) results. Also, CO-RADS group 4 and 5 had 109 patients with negative RT-PCR results. There was no statistically significant difference between total CT-IS and RT-PCR results(Table 5).

There was a statistically significant difference between total CT-IS and distribution of lesions. The mean total CT-IS, in both ventral and dorsal lesions, was statistically significantly increased compared to only ventral lesion or only dorsal lesion (both $P<0.001$ ). The mean total CT-IS for dorsal lesion was statistically higher than the ones for ventral lesion $(P<0.05)$ (Table 6).

Of the participants, 266 (95\%) were admitted to the medical floor, $14(5 \%)$ patients were admitted to the ICU, and 18 (6.4\%) were transferred from the medical floor to the ICU, and. Eighteen (6.4\%) patients died of COVID-19 during hospitalization. Of the CO-RADS group 5 patients, 154 (57\%) were from the medical floor, $11(61 \%)$ were from ICU who were transferred from the medical floor, and 13 (92\%) were from the ICU. There was a statistically significant difference between CT-IS and hospitalization $(P<0.001)$. The mean total CT-IS of all study groups was $6.3 \pm 4.7$. The mean total CT-IS of patients admitted to the medical floor was 5.5 \pm 3.6. The mean total CT-IS of patients admitted to ICU was $16.9 \pm 4.8$. The mean total CT-IS of patients transferred to the ICU from the medical floor was $9.7 \pm 6.8$. The mean total CT-IS of patients admitted to the ICU was remarkably higher than those admitted to the medical floor $(P<0.001)$. Also, the mean total CT-IS of ICU patients transferred from the medical floor was remarkably higher than those remained in the medical floor $(P=0.04)$. The mean total CT-IS of the recovered patients was 5.6 \pm 3.7. The mean total CT-IS of patients died of COVID-19 during the hospitalization was $16.7 \pm 5.5$. The mean total CT-IS was statistically significantly different between patients recovered and died of COVID-19 $(P<0.001)$.

\section{Discussion}

More than 8 million people were infected from COVID-19 and about 450,000 people died of COVID-19 around the world [12]. Early diagnosis is very important for disease control and treatment in COVID-19, which is a highly contagious disease. The RT-PCR assay used as the gold standard diagnostic tool in COVID 19 disease has some limitations, such as: high false-negative results rate, sample collection, insufficient supply of nucleic acid kits, and limited laboratory facilities $[5,13]$. Therefore, chest $\mathrm{CT}$ has become widespread to use as a practical, fast, and reliable investigative tool for diagnosing and evaluating COVID-19 in the outbreak field. CO-RADS was evolved as a categorical system to appraise the suspicion of COVID-19's pulmonary involvement and to provide standard communication on $\mathrm{CT}$ 
Table 4 Computed tomography features between COVID-19 Reporting and Data System (CO-RADS) groups

\begin{tabular}{|c|c|c|c|c|c|c|}
\hline & Total (N: 280) & $\begin{array}{l}\text { CO-RADS } 2 \\
\text { group }(N: 30)\end{array}$ & $\begin{array}{l}\text { CO-RADS } 3 \\
\text { group }(N: 42)\end{array}$ & $\begin{array}{l}\text { CO-RADS } 4 \\
\text { group }(N: 30)\end{array}$ & $\begin{array}{l}\text { CO-RADS } 5 \\
\text { group }(N: 178)\end{array}$ & $P^{*}$ \\
\hline Right upper lobe & $190(67.9)$ & $11(36.7)$ & $9(21.4)$ & $15(50.0)$ & $155(87.1)$ & $<0.001$ \\
\hline Right middle lobe & $157(56.1)$ & $5(16.7)$ & $5(11.9)$ & $11(36.7)$ & $136(76.4)$ & $<0.001$ \\
\hline Right lower lobe & $236(84.3)$ & $15(50.0)$ & $25(59.5)$ & $25(83.3)$ & $171(96.1)$ & $<0.001$ \\
\hline Left upper lobe & $192(68.6)$ & $12(40.0)$ & $6(14.3)$ & $15(50.0)$ & $159(89.3)$ & $<0.001$ \\
\hline Left lower lobe & $218(77.9)$ & $14(46.7)$ & $15(35.7)$ & $20(66.7)$ & $169(94.9)$ & $<0.001$ \\
\hline Ventral & $61(21.8)$ & $13(43.3)$ & 7 (16.7) & $4(13.3)$ & $37(20.8)$ & 0.017 \\
\hline Dorsal & $262(93.6)$ & $22(73.3)$ & $36(85.7)$ & $29(96.7)$ & $175(98.3)$ & $<0.001$ \\
\hline Ventral + dorsal & $43(15.4)$ & $5(16.7)$ & $1(2.4)$ & $3(10.0)$ & $34(19.1)$ & 0.045 \\
\hline Dominant lobe & & & & & & 0.001 \\
\hline Right upper lobe & $35(12.5)$ & $7(23.3)$ & $9(21.4)$ & $4(13.3)$ & $15(8.4)$ & \\
\hline Right middle lobe & $8(2.9)$ & $0(0.0)$ & $2(4.8)$ & $2(6.7)$ & $4(2.2)$ & \\
\hline Right lower lobe & $114(40.7)$ & $8(26.7)$ & $13(31.0)$ & $15(50.0)$ & $78(43.8)$ & \\
\hline Left upper lobe & $25(8.9)$ & $4(13.3)$ & $4(9.5)$ & $3(10.0)$ & $14(7.9)$ & \\
\hline Left lower lobe & $49(17.5)$ & $9(30.0)$ & $13(31.0)$ & $4(13.3)$ & $23(12.9)$ & \\
\hline Two lobes & $49(17.5)$ & $2(6.7)$ & $1(2.4)$ & $2(6.7)$ & $44(24.7)$ & 0036 \\
\hline Right lower + left lower lobes & $25(8.9)$ & $0(0.0)$ & $1(2.4)$ & $1(3.3)$ & $23(12.9)$ & \\
\hline Right upper + left upper lobes & $7(2.5)$ & $1(3.3)$ & $0(0.0)$ & $0(0.0)$ & $6(3.4)$ & \\
\hline Right upper + right lower lobes & $6(2.1)$ & $1(3.3)$ & $0(0.0)$ & $1(3.3)$ & $4(2.2)$ & \\
\hline The others & $11(3.9)$ & $0(0.0)$ & $0(0.0)$ & $0(0.0)$ & $11(6.2)$ & \\
\hline Central & $56(20.0)$ & $13(43.3)$ & $4(9.5)$ & $3(10.0)$ & $36(20.2)$ & 0.002 \\
\hline Peripheral & $270(96.4)$ & $24(80.0)$ & $40(95.2)$ & $30(100.0)$ & $176(98.9)$ & $<0.001$ \\
\hline Ground glass opacity & $221(78.9)$ & $1(3.3)$ & $32(76.2)$ & $22(73.3)$ & $166(93.3)$ & $<0.001$ \\
\hline Consolidation & $108(38.6)$ & $14(46.7)$ & $10(23.8)$ & $12(40.0)$ & $72(40.4)$ & 0.173 \\
\hline Crazy paving & $82(29.3)$ & $0(0.0)$ & $4(9.5)$ & $6(20.0)$ & $72(40.4)$ & $<0.001$ \\
\hline Halo & $101(361)$ & $2(6.7)$ & $11(26.2)$ & $16(53.3)$ & $72(40.4)$ & $<0.001$ \\
\hline Revers halo & $7(2.5)$ & $0(0.0)$ & $2(4.8)$ & $0(0.0)$ & $5(2.8)$ & 0.477 \\
\hline Vascular thickening & $78(27.9)$ & $0(0.0)$ & $4(9.5)$ & $5(16.7)$ & $69(38.8)$ & $<0.001$ \\
\hline Septal thickening & $6(2.1)$ & $1(3.3)$ & $0(0.0)$ & $0(0.0)$ & $5(2.8)$ & 0.541 \\
\hline Pleural thickening & $5(1.8)$ & $1(3.3)$ & $0(0.0)$ & $1(3.3)$ & $3(1.7)$ & 0.661 \\
\hline Subpleural band & $50(17.9)$ & $1(3.3)$ & $0(0.0)$ & $4(13.3)$ & $45(25.3)$ & $<0.001$ \\
\hline Architectural distortion & $72(25.7)$ & $2(6.7)$ & $4(9.5)$ & $3(10.0)$ & $63(35.4)$ & $<0.001$ \\
\hline Vacuolization & $30(10.7)$ & $0(0.0)$ & $2(4.8)$ & $1(3.3)$ & $27(15.2)$ & 0.014 \\
\hline Bronchial wall thickening & $1(0.4)$ & $1(3.3)$ & $0(0.0)$ & $0(0.0)$ & $0(0.0)$ & 0.039 \\
\hline Centrilobular nodules & $26(9.3)$ & $22(73.3)$ & $3(7.1)$ & $1(3.3)$ & $0(0.0)$ & $<0.001$ \\
\hline Air bronchogram & $18(6.4)$ & $5(16.7)$ & $4(9.52)$ & $2(6.7)$ & $7(3.9)$ & 0.052 \\
\hline
\end{tabular}

*Chi-square test

assessment [11]. Also, CT-IS can be helpful as an imaging tool for evaluation of the severity and extent of COVID-19 pneumonia.

In our study, all chest $\mathrm{CT}$ images were categorized into five groups based on the CO-RADS (CO-RADS 2 to CORADS 5). CO-RADS classification of our study patients was compatible with the literature. We found that the CO-RADS system has a remarkable role in diagnosing COVID-19 pneumonia. According to the CO-RADS classification, CO-RADS 5 refers to a very high probability for COVID-19 pneumonia, based on typical CT findings of lung involvement. Besides, CO-RADS 2 refers to a low probability for COVID-19 pneumonia, based on CT findings of lung involvement, which are not compatible with COVID-19 [8]. RT-PCR results were strongly correlated among CO-RADS groups in our study. The number of patients with positive RT-PCR in the CO-RADS group 5 was significantly higher than those in other CO-RADS groups. Prokop et al. [11] showed CO-RADS was powerful to diagnose COVID-19. Also, in our study, all of the CO-RADS 2 group patients had negative RT-PCR results. Regardless of RT-PCR test results, the majority of hospitalized patients were in the 


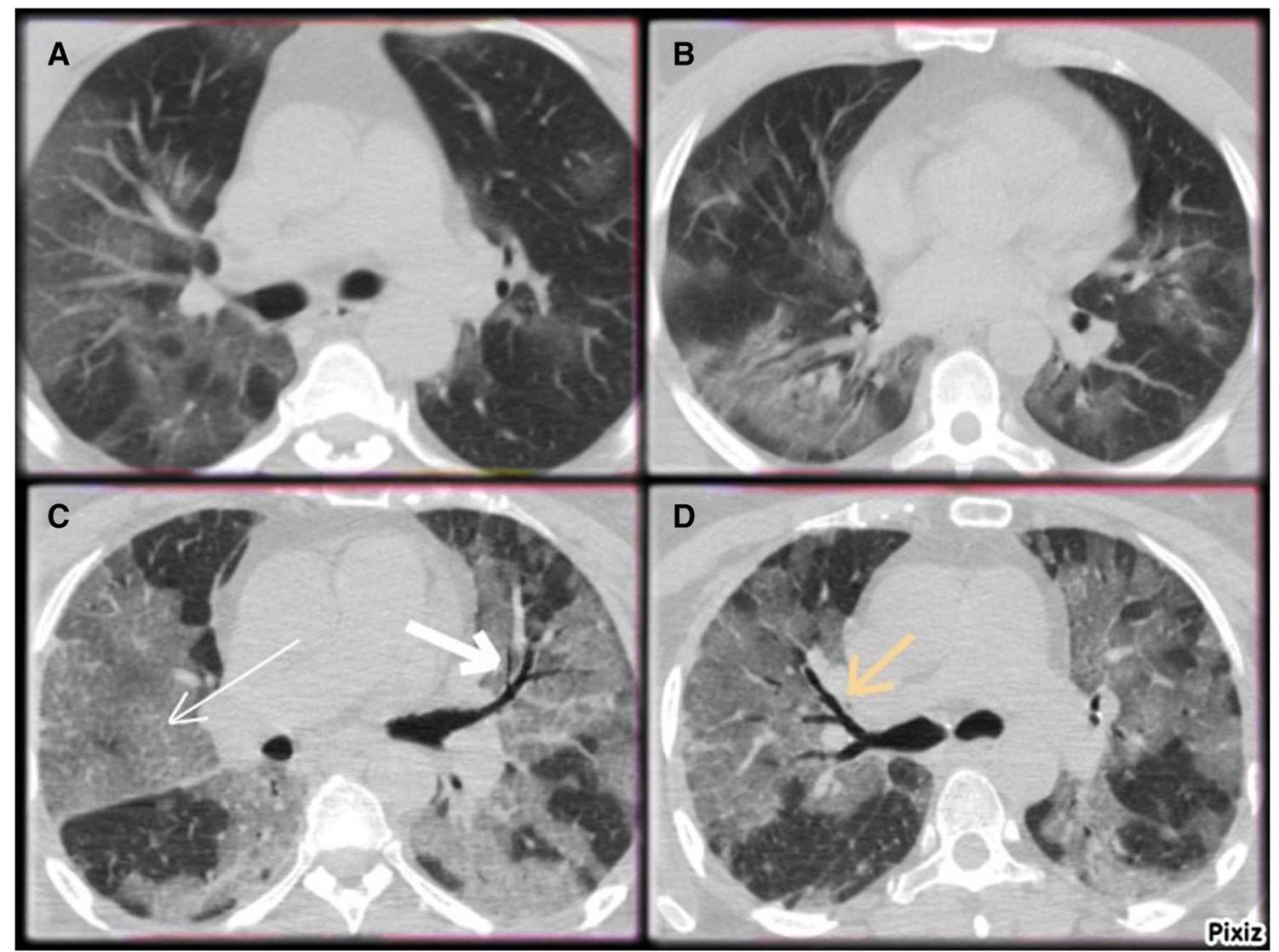

Fig. 3 a/b CO-RADS 5: CT scans of 49-year old male with COVID-19 Widespread bilateral ground-glass opacities (GGO). c/d CO-RADS 5: CT scans of 27-year

Table 5 Computed tomography (CT) features, CT COVID-19 Reporting and Data System (CO-RADS) groups and CT involvement score (CT -IS) between polymerase chain reaction (PCR) results

\begin{tabular}{llllc}
\hline & $\begin{array}{l}\text { PCR- } \\
(N: 169)\end{array}$ & $\begin{array}{l}\text { PCR+ } \\
(N: 111)\end{array}$ & $\begin{array}{l}\text { Total } \\
(N: 280)\end{array}$ & $P^{*}$ \\
\hline Right upper lobe & $107(63.3)$ & $83(74.8)$ & $190(67.9)$ & 0.045 \\
Right middle lobe & $83(49.1)$ & $74(66.7)$ & $157(56.1)$ & 0.004 \\
Right lower lobe & $132(78.1)$ & $104(93.7)$ & $236(84.3)$ & 0.001 \\
Left upper lobe & $109(64.5)$ & $83(74.8)$ & $192(68.6)$ & 0.07 \\
Left lower lobe & $116(686)$ & $102(91.9)$ & $218(77.9)$ & $<0.001$ \\
& & & & $<0.001$ \\
CO-RADS 2 & $30(17.8)$ & $0(0.0)$ & $30(10.7)$ & \\
CO-RADS 3 & $30(17.8)$ & $12(10.8)$ & $42(15.0)$ & \\
CO-RADS 4 & $14(8.3)$ & $16(14.4)$ & $30(10.7)$ & \\
CO-RADS 5 & $95(56.2)$ & $83(74.8)$ & $178(63.6)$ & \\
Ventral & $39(23.1)$ & $22(19.8)$ & $61(21.8)$ & 0.619 \\
Dorsal & $152(89.9)$ & $110(99.1)$ & $262(93.6)$ & 0.005 \\
Ventral+dorsal & $22(13.0)$ & $21(18.9)$ & $43(15.4)$ & 0.242 \\
Mean CT-IS & $6.0 \pm 4.6$ & $6.8 \pm 4.8$ & $6.3 \pm 4.7$ & 0.063 \\
\hline
\end{tabular}

*Mann Whitney $U$, chi-square test
CO-RADS-4 and CO-RADS 5 groups. We showed that even if RT-PCR analysis is negative, CO-RADS can be useful in predicting COVID-19. Xie et al. [14] suggested that positive CT findings of a viral pneumonia may be a strong suspect for COVID-19 even though RT-PCR result was negative. Ai et al. reported a research of 1014 patients who underwent both chest CT and RT-PCR assay for assessment of COVID-19. They suggested that chest CT can be considered as an investigative tool for diagnosis and evaluation in the outbreak field, because positive chest CT findings for COVID-19 had a sensitivity of 97\%. [15]. Lessmann et al. conducted a study by using artificial intelligence for assessment of CO-RADS and Chest CT Severity Score in patients with suspected COVID-19 pneumonia. They found that the system discriminated with high performance between patients with COVID-19 and those without COVID-19 by using CO-RADS and Chest CT Severity Score [16]. Fujioka et al. noticed that CO-RADS maintains remarkable performance and perfect interobserver agreement by using chest CT images for predicting COVID-19 pneumonia [17]. Salehi et al. reported a review of COVID-19 imaging reporting and data system (COVID-RADS) which is a categorical reporting system likewise CO-RADS. They noticed that 
Table 6 Computed tomography (CT) involvement score and clinical outcomes between anatomic distribution of CT features

\begin{tabular}{|c|c|c|c|c|c|}
\hline & Total & Only ventral $(N: 18)$ & Only dorsal ( $N: 219)$ & $\begin{array}{l}\text { Both ventral and } \\
\text { dorsal }(N: 43)\end{array}$ & $P^{*}$ \\
\hline Mean CT-IS & $6.3 \pm 4.7$ & $3.1 \pm 1.7$ & $5.5 \pm 3.6^{\mathrm{A}}$ & $11.8 \pm 6.4^{\mathrm{BC}}$ & $<0.001$ \\
\hline Length of hospital stay (days) & $9.2 \pm 6.1$ & $5.6 \pm 1.0^{\mathrm{BD}}$ & $9.2 \pm 5.3$ & $10.7 \pm 9.8$ & $<0.001$ \\
\hline Duration of symptom (days) & $4.8 \pm 2.7$ & $4.6 \pm 2.6$ & $4.7 \pm 2.7$ & $5.1 \pm 3.0$ & 0.848 \\
\hline PCR & & & & & 0.006 \\
\hline Negative & $169(60.4)$ & $17(94.4)$ & $130(59.4)$ & $22(51.2)$ & \\
\hline Positive & $111(39.6)$ & $1(5.6)$ & 89 (40.6) & $21(48.8)$ & \\
\hline Admission & & & & & $<0.001$ \\
\hline Medical floor & $248(88.6)$ & $18(100.0)$ & $203(92.7)$ & $27(62.8)$ & \\
\hline ICU & $14(5.0)$ & $0(0.0)$ & $4(1.8)$ & $10(23.3)$ & \\
\hline \multirow[t]{2}{*}{$\begin{array}{l}\text { Transferred to ICU from the medi- } \\
\text { cal floor }\end{array}$} & $18(6.4)$ & $0(0.0)$ & $12(5.5)$ & $6(14.0)$ & \\
\hline & & & & & $<0.001$ \\
\hline Survival & $262(93.6)$ & $18(100.0)$ & $214(97.7)$ & $30(69.8)$ & \\
\hline Death & $18(6.4)$ & $0(0.0)$ & $5(2.3)$ & $13(30.2)$ & \\
\hline
\end{tabular}

*Kruskal-Wallis and chi-square test. Post hoc Dunn-Bonferroni test used on parameters with $P<0.05$

${ }^{\mathrm{A}}<0.05$ versus group $\mathrm{D} ;{ }^{\mathrm{B}}<0.001$ versus group $\mathrm{V}$; ${ }^{\mathrm{C}}<0.001$ versus group $\mathrm{VD} ;{ }^{\mathrm{D}}<0.001$ versus group $\mathrm{D} ; P C R$ polymerase chain reaction, $I C U$ intensive care unit

COVID-RADS facilitated the interpretation and reporting of imaging examinations and advanced the quality of patient care [18].

In our study, the extension of pulmonary lesions in the CO-RADS 5 group was significantly greater than the other CO-RADS groups. Likewise, the CO-RADS 5 patients are more likely to have bilateral, multilobe (especially involved in lower lobes), peripheral, and dorsal involvement than the other groups. On the other hand, the involvement of most pulmonary lesions in the CO-RADS 2 was more likely distributed peribronchovascular (central) and ventral. The presence of GGO, crazy paving sign, vascular thickening sign, halo sign, subpleural band, architectural distortion, vacuolization, in the CO-RADS 5 group were strongly higher according to the other groups. The presence of bronchial wall thickening, centrilobular nodules, and tree in the bud in the CO-RADS 2 group was strongly higher according to the other groups. Also, the presence of crazy paving sign, vascular thickening sign, was not observed in CO-RADS 2 group. Bao et al. reported a systematic review which was evaluating the chest CT features in over 2700 COVID-19 patients. Chest CT abnormalities were GGO, pleural thickening, interlobular septal thickening, air bronchogram, respectively; also, were bilateral, had a peripheral distribution, and involved the lower lobes [19].

In our study, CT-IS of all chest CT images was calculated and compared with CO-RADS groups. The mean total CT-IS was strongly correlated between CO-RADS groups. Likewise, the mean total CT-IS in CO-RADS 5 group was remarkably different and higher according to the other CO-RADS groups. The mean total CT-IS was statistically significantly different among hospitalization status. The mean total CT-IS of patients admitted to the ICU was significantly higher than those admitted to the medical floor. Also, the mean total CT-IS of patients transferred to the ICU from the medical floor was significantly higher than those admitted to the medical floor. Also in our study, we found that the mean total CT-IS of patients dead throughout a long hospitalization was strongly different and higher than survivors. We showed that CT-IS accurately identified high-risk patients with COVID-19, which is compatible with the literature. Yang et al. conducted similar research by using a chest CT severity score (CT-SS) for appraising severe COVID-19. They demonstrated that this severity score could be utilized rapidly and practically in COVID-19 screening for assessing the severity of lung involvement [20]. Li et al. noticed that the CT visual quantitative analysis had high compatibility and could project the severity of COVID-19 [21].

\section{Limitations}

This study was conducted in a single center. Also, the number of cases in the CO-RADS groups were not even and relatively small. Additionally, $60.4 \%$ of participants had negative RT-PCR test results. However, the false negative is common for PCR in COVID-19. And this study will contribute to the current literature of COVID-19 in regards to diagnosing and outcome.

In conclusion, this study demonstrated that CO-RADS is useful in detecting COVID-19 pneumonia, even if the RTPCR test is negative. CT-IS can be helpful as an imaging tool for evaluation of the severity and extent of COVID-19 
pneumonia. Moreover, CT-IS has a strong predictive power on patients with COVID-19 outcomes, such as hospital admission.

Author contributions MÖ contributed to conceptualization (equal), data curation (equal), formal analysis (equal), investigation (equal), methodology (equal), resources (equal), software (equal), supervision (equal), validation (equal), visualization (equal), writing-original draft (equal), writing-review and editing (equal); AA and SA were involved in conceptualization (equal), data curation (equal), formal analysis (equal), investigation (equal), methodology (equal), project administration (equal), resources (equal), software (equal), supervision (equal), validation (equal), visualization (equal), writing-original draft (equal), writing-review and editing (equal). All authors approved the final version of the manuscript for publication.

Funding We received no financial support for the research, authorship, and/or publication of this article.

Availability of data and materials Not applicable.

Code availability Not applicable.

\section{Compliance with ethical standards}

Conflict of interest The authors declare that they have no conflict of interest.

Ethics approval The institutional ethical board of the local training and research hospital reviewed and approved this retrospective study (decision date: 28 April 2020, no: 452). Also, we attached the ethical approval in a separate file as a PDF document in your system.

Ethical standard This article does not contain any studies with human participants or animals performed by any of the authors.

Consent to participate Not applicable.

Consent for publication Not applicable.

\section{References}

1. Lu H, Stratton CW, Tang YW (2020) Outbreak of pneumonia of unknown etiology in Wuhan, China: the mystery and the miracle. J Med Virol 92:401-402. https://doi.org/10.1002/jmv.25678

2. Xu X, Chen P, Wang J et al (2020) Evolution of the novel coronavirus from the ongoing Wuhan outbreak and modeling of its spike protein for risk of human transmission. Sci China Life Sci 63:457-460. https://doi.org/10.1007/s11427-020-1637-5

3. Naming the coronavirus disease (COVID-19) and the virus that causes it. https://www.who.int/emergencies/diseases/novel-coron avirus-2019/technical-guidance/naming-the-coronavirus-disease(covid-2019)-and-the-virus-that-causes-it. Accessed 21 June 2020

4. Fda Where can I go for updates and more information? Fact sheet for patients CDC-2019-nCoV real-time RT-PCR diagnostic panel. https://www.fda.gov/media/134921/download. Accessed 5 Aug 2020

5. Yang Y, Yang M, Shen C et al (2020) Evaluating the accuracy of different respiratory specimens in the laboratory diagnosis and monitoring the viral shedding of 2019-nCoV infections. MedRxiv. https://doi.org/10.1101/2020.02.11.20021493
6. Lu C, Yang W, Hu Y et al (2020) Coronavirus Disease 2019 (COVID-19) pneumonia: early stage chest CT imaging features and clinical relevance. SSRN Electron J. https://doi.org/10.2139/ ssrn.3543606

7. Fang Y, Zhang H, Xie J et al (2020) Sensitivity of chest CT for COVID-19: comparison to RT-PCR. Radiology. https://doi. org/10.1148/radiol.2020200432

8. Rubin GD, Ryerson CJ, Haramati LB et al (2020) The role of chest 1maging in patient management during the COVID-19 pandemic. Chest. https://doi.org/10.1016/j.chest.2020.04.003

9. Pan F, Ye T, Sun P et al (2020) Time course of lung changes at chest CT during recovery from Coronavirus Disease 2019 (COVID-19). Radiology 295:715-721. https://doi.org/10.1148/ radiol.2020200370

10. Zhao W, Zhong Z, Xie X et al (2020) Relation between chest CT findings and clinical conditions of Coronavirus Disease (COVID19) pneumonia: a multicenter study. Am J Roentgenol 214:10721077. https://doi.org/10.2214/AJR.20.22976

11. Prokop M, van Everdingen W, van Rees VT et al (2020) CORADS - a categorical CT assessment scheme for patients with suspected COVID-19: definition and evaluation. Radiology. https ://doi.org/10.1148/radiol.2020201473

12. World Health Organization. Coronavirus disease (COVID-19) Situation Report-150. Available from: https://www.who.int/docs/ default-source/coronaviruse/situation-reports/20200618-covid-19sitrep-150.pdf?sfvrsn=aa9fe9cf_4. Accessed 5 Aug 2020

13. Xiao SY, Wu Y, Liu H (2020) Evolving status of the 2019 novel coronavirus infection: proposal of conventional serologic assays for disease diagnosis and infection monitoring. J Med Virol 92:464-467. https://doi.org/10.1002/jmv.25702

14. Xie X, Zhong Z, Zhao W et al (2020) Chest CT for typical 2019nCoV pneumonia: relationship to negative RT-PCR testing. Radiology. https://doi.org/10.1148/radiol.2020200343

15. Ai T, Yang Z, Hou H et al (2020) Correlation of chest CT and RTPCR testing in Coronavirus Disease 2019 (COVID-19) in China: a report of 1014 cases. Radiology. https://doi.org/10.1148/radio 1.2020200642

16. Lessmann N, Sánchez CI, Beenen L et al (2020) Automated assessment of CO-RADS and chest CT severity scores in patients with suspected COVID-19 using artificial intelligence. Radiology. https://doi.org/10.1148/radiol.2020202439

17. Fujioka T, Takahashi M, Mori M et al (2020) Evaluation of the usefulness of CO-RADS for chest CT in patients suspected of having COVID-19. Diagnostics 10:608. https://doi.org/10.3390/ diagnostics 10090608

18. Salehi S, Abedi A, Balakrishnan S, Gholamrezanezhad A (2020) Coronavirus disease 2019 (COVID-19) imaging reporting and data system (COVID-RADS) and common lexicon: a proposal based on the imaging data of 37 studies. EurRadiol 30:4930-4942. https://doi.org/10.1007/s00330-020-06863-0

19. Bao C, Liu X, Zhang H et al (2020) Coronavirus Disease 2019 (COVID-19) CT findings: a systematic review and meta-analysis. J Am CollRadiol. https://doi.org/10.1016/j.jacr.2020.03.006

20. Yang R, Li X, Liu H et al (2020) Chest CT severity score: an 1maging tool for assessing severe COVID-19. RadiolCardiothorac Imaging 2:e200047. https://doi.org/10.1148/ryct.2020200047

21. Li K, Fang Y, Li W et al (2020) CT image visual quantitative evaluation and clinical classification of coronavirus disease (COVID19). EurRadiol. https://doi.org/10.1007/s00330-020-06817-6

Publisher's Note Springer Nature remains neutral with regard to jurisdictional claims in published maps and institutional affiliations. 